\title{
Inhibitors of lactate dehydrogenase (hLDH5) from Polygala flavescens subsp. flavescens
}

Marinella De Leo ${ }^{1}$, Lorenzo Peruzzi ${ }^{1}$, Carlotta Granchi ${ }^{1}$, Tiziano Tuccinardii ${ }^{1}$, Filippo Minutolo ${ }^{1}$, Nunziatina De Tommasi ${ }^{3}$, Alessandra Braca $^{1}$

${ }^{1}$ Dipartimento di Farmacia, Università di Pisa, via Bonanno 6 and 33, 56126 Pisa, Italy

2 Dipartimento di Biologia, Università di Pisa, via Derna 1, 56126 Pisa, Italy

${ }_{3}^{3}$ Dipartimento di Farmacia, Università degli Studi di Salerno, via Giovanni Paolo II 132, 84084 Fisciano (SA), Italy

The human isoform 5 of lactate dehydrogenase ( $h$ LDH5) is an enzyme up-regulated in tumor tissues since cancer cells depend mainly on anaerobic respiration and their glycolytic rate is up to 200 times higher than that of the normal tissue. hLDH5 inhibition should cause cancer cell death by starvation, without interfering with healthy cells that normally use oxidative phosphorylation for ATP generation. Inhibition of LDH is so considered as a promising target in cancer treatment, since it is possible to cause a starving of cancerous cells by reducing glycolysis or by inhibiting the conversion of glucose to lactate. In the course of our research program on the hLDH5 inhibitory activity of natural products [1], a chemical study of $P$. flavescens subsp. flavescens was carried out. Polygala L. genus (Polygalaceae) is well known to contain phenolic oligosaccharides, xanthones, lignans, and triterpenic saponins and it's largely used in the traditional medicine [2]. Ten new compounds were isolated from the $n$ - $\mathrm{BuOH}$ residue of the aerial parts through Sephadex and RP-HPLC separations, including four flavonol glycosides, two oligosaccharides, one $\alpha$-ionone, and three triterpenoidic saponins, together with two known oligosaccharides and two flavonol glycosides. All structures were elucidated on the basis of their spectroscopic and spectrometric data. The isolates were assayed for their inhibitory activity against $h$ LDH5 and 3,6'-di- $O$-sinapoylsucrose showed an $\mathrm{IC}_{50}$ value of $90.4 \mu \mathrm{M}$. Modeling studies were carried out to suggest the putative interaction mode of this compound in the enzyme active site. This analysis highlighted that 3,6'-di- $O$ sinapoylsucrose shows a high number of $\mathrm{H}$-bonds and interacts with enzyme regions rarely explored by the known $h$ LDH5 inhibitors.

[1] Bader A, Tuccinardi T, Granchi C, Martinelli A, Macchia M, Minutolo F, De Tommasi N, Braca A. Phytochemistry 2015; 116: 262-268.

[2] Klein LC Jr, Faloni de Andrade S, Cechinel Filho V. Chem. Biodivers. 2012; 9: 181-209. 